\title{
Architectural Mismatch in Service-Oriented Architectures
}

\author{
Kevin Bierhoff \\ Institute for Software Research, Carnegie Mellon University \\ 5000 Forbes Avenue, Pittsburgh, PA 15213 \\ kevin.bierhoff @ cs.cmu.edu
}

\author{
Mark Grechanik and Edy S. Liongosari \\ Systems Integration Group, Accenture Technology Labs \\ 161 North Clark Street, Chicago, IL 60601 \\ \{mark.grechanik, edy.s.liongosari\}@accenture.com
}

\begin{abstract}
Architectural mismatch results from implicit and conflicting assumptions that designers of components make about the environments in which these components should operate. While architectural mismatch was extensively studied in monolithic and distributed applications, it has not been applied to Service-Oriented Architectures (SOAs).

A major contribution of this paper is the analysis of how architectural mismatch affects SOAs. We study how implicit and conflicting assumptions that designers make about web services and their compositions affect the quality of resulting SOA-based systems. We support our analysis with empirical data that we collected from a large-scale SOA-based project within Accenture and other smaller projects.
\end{abstract}

\section{Introduction}

Architectural mismatch results from implicit and conflicting assumptions that designers of components make about the environment in which these components will operate [9]. Architectural mismatch impedes constructing applications from third-party reusable components that, on a superficial level, appear compatible. Even if components are written in the same programming language, run on the same platform, and are intended for reuse, software engineers can encounter significant problems in getting components to work together. Engineers may have to re-implement existing functionality, provide glue code to mediate between components, or even change component implementations in order to overcome mismatch. The resulting systems can be intolerably large and slow [9].

As a concrete example, Garlan et al observed a mismatch between message data models of two components they used in constructing an interactive modeling environment. One component assumed that messages would be passed as heap data structures while another component expected character strings. Even though this mismatch was discovered early, non-trivial message conversions were necessary to make the two components interoperable. The conversion routines represented a significant engineering effort and seriously affected the performance of the resulting system [9]. This paper will show that this and other problems of architectural mismatch are still and sometimes more relevant in developing Service-Oriented Architectures.

Service-Oriented Architectures (SOAs) define how software components called services are organized into structures to support business requirements [12]. Web services are software components that exchange information (i.e., interoperate [2]) in heterogenous environments including the Internet. They currently gain widespread acceptance partly because of the business demand for applications to exchange information [8]. SOAs and web services enable organizations to automate business processes by increasing the speed and effectiveness of information exchange.

Architectural mismatch offers a taxonomic framework for understanding challenges in building applications out of re-usable components. This framework was extensively studied for monolithic and distributed applications; however, it has not been applied to SOAs. A major contribution of this paper is our analysis of how architectural mismatch affects SOAs. We study how implicit and conflicting assumptions that designers make about web services and their compositions affect the quality of resulting SOA-based systems. We support our analysis with empirical data that we collected as part of Tarpon, a large-scale SOA-based project within Accenture, and other smaller projects.

We show that architectural mismatch is not only helpful in categorizing and understanding practical challenges in building SOAs. It turns out that all originally described constituents of architectural mismatch are still relevant in the context of SOA. Two primary concerns for architectural mismatch in SOAs are messaging overhead and incompatibilities between SOA platform vendors.

\section{Categories of Architectural Mismatch}

Architectural mismatch provides a taxonomic framework for understanding how conflicting assumptions arise. 
The following categorization of causes for architectural mismatch is based on Garlan et al's original taxonomy [9].

1. Assumptions about the nature of components can be divided into four sub-categories.

- Functionality supply. Components provide functionality that may not be needed in the final assembly, leading to excessive code size of resulting applications.

- Infrastructure expectations. Components may assume the presence of certain resources (e.g., libraries or hardware) that may not be available, rendering these components non-usable.

- Control model. Designers assume that their components will own the main thread of control that contains an infinite event processing loop. Coordinating these event loops is non-trivial and may require change to component implementations.

- Data manipulation. Designers make assumptions about how clients manipulate component data structures.

2. Assumptions about communication between components can be divided into two sub-categories.

- Asynchronous communication. Asynchronous messaging can force conceptually singlethreaded applications to be implemented with multiple threads.

- Message data model. Incompatibilities in the formats of messages that components exchange can lead to massive performance overhead due to costly message conversions.

3. Global architecture structure. Designers assume that different clients of a component operate independently. However, clients may delegate tasks to each other, violating the independence assumption. Such dependencies may be subtle, for example, when two components access the same resource.

4. Construction process. Designers assume an order in which components should be constructed and how these components are combined into the system. Conflicting construction order assumptions can complicate the application's construction process.

The following four section discuss how these categories of architectural mismatch affect SOAs. Afterwards we summarize empirical evidence for our findings.

\section{The Nature of Services}

From an architectural point of view, a service provides a logically coherent piece of functionality to its clients. Mismatch can occur when designers of services define their nature in ways that make it difficult to use these services.

\subsection{Functionality Supply}

Problems with functionality supply exist in SOA both for individual services and because of the employed SOA infrastructure. Individual services are designed to be reusable and will therefore provide a certain flexibility in the way they can be used. This can lead to oversized services with bloated interfaces. For example, an interest payment calculation service could be used for home mortgages, auto and credit card loans. The rules of calculation are somewhat different for different loan-types. Thus, the service's incoming and outgoing messages now have to include the type of loan as well as different regulatory and location parameters needed for interest calculations, regardless of whether clients actually exercise this flexibility. Thus mismatch in functionality supply can lead to oversized messages.

Another source of mismatch in functionality of individual services is the level of service granularity of an SOA. While many SOA experts advocate using business processes to define the scope and granularity of the underlying services, the problem of granularity mismatch does not go away because business processes can often be decomposed into smaller process steps. If processes are too fine-grained then messaging overhead becomes overwhelming. If services are too large then messages can become big and cause services to respond slowly.

This messaging overhead directly impacts the performance of the service. Studies have shown that XML messages are typically 10 to 50 times larger than their binary counterparts and that XML-related tasks such as parsing, transformation and serialization consumed over $93 \%$ of total processing of typical XML documents [4]. Thus, a single highly used service with oversized messages can impact an entire SOA infrastructure.

Additionally, SOA middleware commonly addresses non-functional concerns such as security. In order to address these concerns, the middleware commonly expects that services expose certain interfaces. However, despite having standards like WS-Security, these interfaces may differ between middleware vendors, leading to vendor lockin or mismatches in critical areas such as security or reliability (see section 7.1.1). While mismatches between individual services could potentially be corrected by changing service implementations, mismatches between SOA middleware implementations from different vendors cannot be addressed directly. 


\subsection{Infrastructure Expectation}

Many traditional component technologies use late binding, i.e. components are not connected until they are executed. Essentially, lately bound components only depend on the interfaces of other components and not on their implementation. This introduces additional flexibility, in particular the ability to change the implementation of one component without the need to re-compile other components. On the other hand, late binding causes brittleness if a component disappears that other components depend on.

In traditional component development, early binding is a prevalent way to couple components at compile time. Because independence of services is highlighted in the context of SOA we suspect that changing service interfaces and service disappearance may be a more common phenomena in SOAs. Loose coupling between services is one of the promises and advantages of SOA but it requires more work in controlling the dependencies between services and across versions of a service. Service directories currently begin to address this issue.

\subsection{Control Model}

In SOAs, services operate autonomously and typically run in independent processes. Therefore, competition for a main thread of control (as found to be a problem with traditional components) is not a significant issue for services in SOAs. However, SOAs typically orchestrate services in a workflow-like manner using an orchestration engine. The orchestration engine owns the "logical" thread of control that drives the overall application. Control model mismatches can occur when multiple orchestration engines have to cooperate. In particular, orchestration engines may have to cooperate in a peer-to-peer manner across organizational boundaries to accomplish a task (see section 7.1.2). Similar problems can occur in SOAs with a federated topology where orchestrated services are exposed as "logical" services to other orchestration engines, thus creating a hierarchy of orchestrations.

\subsection{Data Manipulation}

On the surface, data manipulation problems are less problematic in SOA than with traditional components. Services typically cannot expose internal data to clients and therefore clients cannot manipulate this data directly. However, if services are stateless, the entire conversational state may be required to be sent back and forth between client and service, thereby effectively exposing internal data to external clients. Clients may only be allowed to modify certain parts of the conversational state received from the service.
In carelessly implemented services, unexpected manipulations of conversational state by clients could lead to inconsistent data and ultimately service malfunctioning. Thus mismatch due to restrictions on data manipulation by clients does exist and can lead to various problems as described in section 7.2.

\section{Communication Between Services}

Communication between applications and services participating in an SOA is typically handled by messaging middleware. Messages are commonly exchanged in XML format and routed through a message bus that connects to all applications and services. Messaging follows an asynchronous model and some services will even publish notification messages without knowing which other services receive these notifications.

\subsection{Asynchronous Communication}

In theory, services in SOA should be autonomous and communicate asynchronously. Asynchronous communication can be a significant complication because seemingly single-threaded applications are forced into multi-threaded implementations. On the positive side, service orchestration engines are specifically designed to facilitate taking advantage of asynchronous communication without burdening developers with traditional problems of multi-threaded software. Unfortunately, services are rarely completely autonomous and they do not communicate solely through asynchronous means. Such deviations from the "norm" can lead to significant problems in properly orchestrating services: orchestration engines and other services have to be prepared for service dependencies and occasional synchronous communication.

\subsection{Message Data Model}

Services typically communicate through XML and therefore require support for accessing and manipulating XML. XML messages are difficult to parse and relatively verbose. Thus messaging overhead is a considerable factor in designing an SOA even if no mismatch occurs [7].

Mismatch in message data models essentially means that messages from one service do not fit the expectations of another service. Consider the case where output of one service is used as part of the input to another service. If the two services use different message formats then the first service's output needs to be converted so that it meets the second service's expectations (see section 7.2).

It has been pointed out that such conversions quickly become a performance bottleneck in SOAs [7]. This is because XML is not only difficult to parse and therefore dif- 
ficult to convert (see section 3.1) but also because the stateless model of services requires messages to carry the entire conversational state, leading to very large messages. Therefore it appears that mismatch in message data models can become a serious problem in SOA-based infrastructures.

\section{Global Architecture Assumptions}

Services, like traditional components, likely assume that their clients are independent from each other. But if a service delegates part of its work to another service and both access a third service then that third service's independence assumption is violated. Such dependencies can be subtle because they might be encoded in the orchestration mechanism rather than the participating services. In this case services do not even know which other services work on tasks they delegated.

\section{Construction Process}

At first glance it appears that mismatching component assumptions about the construction process are eliminated in SOA. This is because services are developed and built independently. By "building" we mean compiling and linking the service implementation. While the situation is certainly much better than with traditional components it appears that managing the build process can still be difficult because services can depend on the interface of other services.

Consider the case where service A depends on service B. One way of building service A is to obtain B's interface definition and use it for generating glue code for communicating with B. If B (directly or indirectly) happens to depend on A's interface as well then it can be challenging to build A and B fully automatically.

In SOA there is another complication: Services also have to be deployed to on a SOA middleware in order to make them available. This can complicate testing of independently constructed services when an expected service is not (yet) available. Techniques similar to unit testing are needed that essentially simulate the presence of other services with "mock services".

\section{Empirical Evidence}

In this section, we provide empirical evidence and show models that describe how architectural mismatch surfaces in SOAs. We review the issues of architectural mismatch in an Accenture project and in message exchanges between services. The section primarily illustrates mismatches in the nature of services and in communication.

\subsection{Tarpon}

Project Tarpon is an SOA R\&D initiative within Accenture to explore the promises and pitfalls of SOA. The initiative will test everything from security to performance, delivering a realistic evaluation of the feasibility and benefits of separating cross-enterprise processes from applications that implement the processes.

\subsubsection{Basic Messaging}

As the first step in this evaluation process, Tarpon performed a series of basic messaging interoperability tests across select SOA platforms from vendors like Microsoft, IBM and Oracle. The tests we performed are a superset of what was published by the WS-Interoperability organization [1]. The idea is to measure the effectiveness of various web services standards such as SOAP, XML and WSDL in alleviating the differences of communication protocols across multiple platforms [14].

We found that even with these established standards, cross-platform interoperability continues to be a challenge. Specifically, we found that:

- different vendors are adopting standards at different pace;

- different vendors are adopting different parts of a standard, and

- complex dependencies among standards further exacerbate confusion in implementation.

Speed of adopting standards. Because standardization is typically a multi-year process, by the time a specification becomes a standard, that standard may be succeeded by a new and better specification. That is exactly what happened to WS-Reliability, a reliable messaging standard that is published by OASIS. By the time it became a standard it was already superseded by a new-and by many measures superior-specification: WS-ReliableMessaging (WS-RM).

The vendors are left with a dilemma of which one to adopt: a published standard or a better specification. To make things worse, the two are completely incompatible. While it is not surprising that some vendors chose to implement the published standard, those that chose the better specification worked hard to ensure that the specification becomes a standard. In the meanwhile, this left users baffled as they had to absorb this incompatibility at their own expense.

Partial adoption of standards. WS-Security is another standard from OASIS that addresses, among other concerns, encryption and authentication of messages. As a fairly complicated standard it contains multiple parts, some of which 
are optional. For example, WS-Security specifies a number of authentication protocols such as username-password pair, X.509, Kerberos and SAML. Except for the usernamepassword pair, it is up to the vendor to choose which other protocols to implement.

This implies that while two products can claim that they support one standard, WS-Security, they may implement two different sets of protocols. For example, one vendor supports Kerberos and the other supports SAML. This complicates cross-platform interoperability. Users have to be aware of these variations and are often times left with the least common denominator.

Complex dependencies among standards. Many standards do not stand alone. They depend on other existing standards. Take for example X.509, a decade-old standard for public key infrastructure published by International Telecommunication Union (ITU). One of its essential components is the key certificate. The format of this certificate is defined by other standards such as RSA Data Security's Public Key Cryptography Standard (PKCS). There are many versions of PKCS-most notably PKCS \#8 and \#12. While it is possible to convert one certificate format to another, the process is non-trivial [11].

As each standard evolves independently, keeping track of the dependencies and compatibilities is a serious and timeconsuming exercise. The situation is worsened by the fact that the number of standards organizations involved in this area continues to grow due to the popularity of web services and SOA. For example, the World Wide Web Consortium, OASIS, Liberty Alliance, Internet Engineering Task Force, Data Management Task Force are all involved in standardizing security and identity management. As such, it is inevitable that they will create multiple conflicting standards.

\subsubsection{Control Model}

Business process orchestration is a key component of SOA that distinguishes SOA from JBOWS (just a bunch of web services). The idea is that each service in SOA is autonomous and message exchanges across services are handled through one or more orchestration engines in a workflow-like manner. This way, service invocation is entirely controlled by orchestration engines.

We found that this control model is too constraining for cross-enterprise scenarios. Take for example electronic prescribing: a doctor creates an electronic prescription. Before a prescription can be forwarded to a pharmacy, it has to go through several organizations including the insurance company that checks for drug coverage under an insurance plan. Since there are many independent organizations involved, it is not clear where service orchestration should reside and how the invocation of services should be strung together.

This calls for an event-driven business process orchestra- tion (or event-driven architecture) [6]. The idea is to move from a static control flow structure-similar to what is typically found in a console program-to an event-based flow as found in many GUI environments. We found that many SOA platforms today do not support this type of control model.

\subsection{Errors In Exchanging XML Data}

It is conservatively estimated that the cost of programming errors in component interoperability just in the capital facilities industry ${ }^{1}$ in the U.S. alone is $\$ 15.8$ billion per year. A primary driver for this high cost is fixing flaws in incorrect data exchanges between interoperating components [3]. An instance of this problem is described in a case study of a large-scale project conducted at KLA-Tencor Corp. [10].

We use the model shown in Figure 1 to describe how services interoperate. In this model, $\mathrm{J}$ and $\mathrm{C}$ are services that interact using XML data $D_{2}$. Service $J$ reads in data $D_{1}$, modifies it, and passes it as data $D_{2}$ to $C$. Service $C$ reads in the data $D_{2}$ expecting it to be an instance of some schema $S$. Since $J$ outputs data $D_{2}$ before $C$ accesses it, concurrency is not relevant. However, because of design or programming errors, service $J$ outputs the data $D_{2}$ as an instance of a different schema $S^{\prime}$, which is not explicitly stated in any design documents. Since $S^{\prime}$ is different from $S$, a runtime error may be issued when $C$ reads in $D_{2}$.

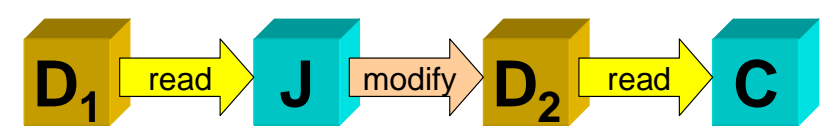

Figure 1. A model of service interoperability.

There are different reasons why programmers make such mistakes when they write the services $\mathrm{J}$ and $\mathrm{C}$. Based on our participation in large-scale projects, we observe that programmers often make wrong assumptions about schemas. Given that many industrial schemas contain thousands of elements and types, it is easy to make mistakes about names of elements and their locations in schemas. The other source of errors lies in the complexity of platform API calls that programmers use to access and manipulate XML data. XML parsers export dozens of different API calls, and mastering them requires a steep learning curve.

Programmers often lack the knowledge of the impact caused by changing the code of some component on other components that interoperate using XML data. This lack of knowledge is an effect of Curtis' law that states that application and domain knowledge is thinly spread and only one or two team members may possess the full knowledge of a

\footnotetext{
${ }^{1} \mathrm{~A}$ capital facility is a structure or equipment which generally costs at least $\$ 10,000$ and has a useful life of ten years or more.
} 
software system [5]. The effect of this law combined with the difficulty of comprehending large-scale XML schemas and high complexity of platform API calls result in components producing XML data that is incompatible for use by other components.

The other source of errors is the disparity in evolving XML schemas and components. Database administrators usually maintain schemas, and programmers maintain components that interoperate using XML data that should be instances of these schemas. If a database administrator modifies a schema and does not inform all programmers whose services are affected by this change then some services will keep modifying XML data according to the obsolete schema.

The problem of mismatch between XML data and schemas is typically addressed by using schema validators that are parts of many XML parsers. In our model shown in Figure 1, an XML parser can validate that the data $D_{2}$ is an instance of the schema $S$ when $J$ produces this data. If the data is not an instance of this schema, then the parser throws a runtime exception. Obviously, it is better to predict possible errors at compile time rather than to deal with them at runtime.

In reality, the situation is even more complicated. Using schemas for validating XML data is often not attempted because it degrades components performance $[15,13]$ and it leads to exceptions when there may not be any problems when processing the data. It is important to know what data elements services $\mathrm{J}$ and $\mathrm{C}$ access and modify, and if no data element accessed by $\mathrm{C}$ is modified by $\mathrm{J}$, then $\mathrm{J}$ and $\mathrm{C}$ may still interact safely even if the data $D_{2}$ is not an instance of the given schema $\mathrm{S}$.

\section{Conclusions}

This paper systematically analyzes how the different categories of architectural mismatch affects Service Oriented Architectures (SOAs). We study how implicit and conflicting assumptions that designers make about web services and their compositions affect the quality of resulting SOAbased systems. We support our analysis with empirical data that we collected as part of Tarpon, a large-scale SOA-based project within Accenture and other smaller projects. We show that architectural mismatch is not only helpful in categorizing and understanding practical challenges in building SOAs. It turns out that all originally described constituents of architectural mismatch are still relevant in the context of SOA. Two primary concerns for architectural mismatch in SOAs are messaging overhead and incompatibilities between SOA middleware vendors.

This paper suggests that architectural mismatch is a promising framework for categorizing and understanding challenges around building SOAs. It provided initial em- pirical evidence for some of the identified causes for architectural mismatch. Future work includes at least three directions. (1) Identification of additional causes for architectural mismatch in SOAs. (2) More complete empirical evidence to understand when mismatch occurs in practice. (3) Mitigating individual causes for architectural mismatch. We mentioned a number of immediate research possibilities throughout the paper. More long-term we hope that understanding the causes for architectural mismatch helps guiding SOA research and practice in their efforts.

Acknowledgements The first author was supported in part by NASA cooperative agreement NNA05CS30A, NSF grant CCF0546550, and Army Research Office grant number DAAD19-021-0389 entitled "Perpetually Available and Secure Information Systems".

\section{References}

[1] Web services interoperability organization website. http://www.ws-i.org/.

[2] IEEE Standard Computer Dictionary: A Compilation of IEEE Standard Computer Glossaries. Institute of Electrical and Electronics Engineers, January 1991.

[3] Cost Analysis of Inadequate Interoperability in the U.S. Capital Facilities Industry, GCR 04-867. NIST, Aug. 2004.

[4] Solving the Very Large Messaging Problem in the Enterprise. www.zapthink.com/report.html?id=WP-0137, February 2005.

[5] B. Curtis, H. Krasner, and N. Iscoe. A field study of the software design process for large systems. Commun. ACM, 31(11):1268-1287, 1988.

[6] V. Dheap and P. A. S. Ward. Event-driven response architecture for event-based computing. In CASCON, pages 70-82, 2005.

[7] T. Erl. Service-Oriented Architecture: A Field Guide to Integrating XML and Web Services. Prentice Hall, 2004.

[8] C. Ferris and J. A. Farrell. What are web services? Commun. ACM, 46(6):31, 2003

[9] D. Garlan, R. Allen, and J. Ockerbloom. Architectural mismatch or why it's hard to build systems out of existing parts. In ICSE, pages 179-185, 1995.

[10] M. Grechanik, D. S. Batory, and D. E. Perry. Design of largescale polylingual systems. In ICSE, pages 357-366, 2004.

[11] A. K. Lenstra and B. de Weger. On the possibility of constructing meaningful hash collisions for public keys. In ACISP, pages 267-279, 2005.

[12] J. McGovern, O. Sims, A. Jain, and M. Little. Enterprise Service Oriented Architectures: Concepts, Challenges, Recommendations, section 1, pages 1-11. The Enterprise Series. Springer, first edition, 2006.

[13] J. Meier, S. Vasireddy, A. Babbar, and A. Mackman. Improving .NET application performance and scalability. Microsoft Corporation, 2004.

[14] E. Newcomer. Understanding Web Services: XML, WSDL, SOAP, and UDDI. Addison-Wesley Professional, May 2002.

[15] R. Schmelzer. Breaking XML to optimize performance. ZapThink LLC - special to SearchWebServices.com, Oct. 2002. 